\title{
A promising strategy against SARS-CoV-2 infected patients: Antisense therapy
}

\section{Hasan Cubuk ${ }^{1 *}$}

${ }^{1}$ Department of Molecular Biology and Genetics, Istanbul Arel University, 34537, Buyukcekmece, Istanbul.

*Corresponding author: Department of Molecular Biology and Genetics, Istanbul Arel University, 34537, Buyukcekmece, Istanbul.

e-mail: hasan.cubk@hotmail.com

\begin{abstract}
As of July 25-2020, 643,412 people in more than 215 countries have been victims of the new type of coronavirus, SARS-CoV-2. Thereby, there is a huge effort to develop a strategy to treat, and or prevent people from SARS-CoV-2 infection. Those efforts could be mainly categorized as drug repurposing, antiSARS-CoV-2 antibodies from people who recovered, and vaccines. However, there is currently no specific treatment available against SARS-CoV-2 infected patients. That's why many new approaches and ideas are still studied every day for the treatment of SARS-CoV-2 infected patients. Antisense therapy is one of these promising approaches to target SARS-CoV-2 genomic RNA specifically and inhibit its activity upon incorrect viral RNA processing. In this study, antisense oligonucleotide (ASO) candidates targeting SARSCoV-2 genomic RNA were designed. High-scored ASOs with a high potential to inhibit SARS-CoV-2 replication and transcription by inducing cleavage of the viral genomic were determined among ASO candidates. For the future, those promising ASOs can be synthesized followed by required modifications and test on SARS-CoV-2 infected Vero cells to screen their efficacy for the treatment of SARS-CoV-2 infected patients.
\end{abstract}

Keywords: antisense oligonucleotides (ASOs); antisense RNA therapy; drug development; SARS-CoV2; In silico

Abbreviations: SARS-CoV-2, Severe acute respiratory syndrome coronavirus-2; ACE2, Angiotensin-converting enzyme 2; ASOs, antisense oligonucleotides; 5Me-dC, 5-methyl deoxycytosine; 7-deaza-dG, deaza G; 2'-O-MOE-RNA, 2'-O-methoxyethyl 5me Uridine; PS, phosphorothioate linkage.

\section{Introduction}

SARS-CoV-2 (severe acute respiratory syndrome coronavirus-2) is a type of single-stranded RNA virus that infects mammals and birds. SARS-CoV-2 utilizes spike (S) proteins in the outer surface to attach host cells through the angiotensin-converting enzyme 2 (ACE2) receptors which mainly on the surface of epithelial cells of the pulmonary alveolus. On the virus side, the RBD domain located on Spike (S) protein is responsible for the host attachment. (Hoffmann et al., 2020; Walls et al., 2020; Yan et al., 2020). Once the viral attachment occurs, viruses enter into the host cells, following endocytosis pathways or direct 
fusion. The viral RNA genome is then released in the cytoplasm. Positive-sense single-stranded viral genome is then directly translated by the host ribosomes without any additional modifications or process. At this point, viral particles are formed through viral RNA translation, followed by a new virion assembly (Fung and Liu, 2019; Jeong et al., 2020; Shang et al., 2020).

COVID-19 caused by SARS-CoV-2 infection has affected over 15,971,347 people and killed 643,412 in more than 215 countries as of July 25, 2020 (Worldometer, 2020). Therefore, there is an urgent need for a relevant treatment strategy. However, there is currently no specific treatment available against SARSCoV-2 infected patients. For this purpose, several repurposing antiviral drugs such as remdesivir, favipiravir, hydroxychloroquine, and lopinavir have been applied around the world to treat SARS-CoV-2 infected patients (Cubuk and Ozbil, 2020; Dhama et al., 2020; Smith et al., 2020). However, drug repurposing studies are a short-term effort to fulfill an urgent need. That's why it cannot help find effective treatments for the SARS-CoV-2 infection. Many efforts have been also directed to develop anti-SARSCoV-2 antibodies from people who recovered to treat those patients. Besides, many institutions and companies have a very big effort to develop efficient vaccines to provide protective immunity to the world population as well (Barrey et al., 2020; Dhama et al., 2020).

On the other site, antisense therapy could be a choice to specifically treat SARS-CoV-2 infection among other treatment strategies; vaccine, antiviral drug repurposing, and anti-SARS2-CoV-2 antibody approach (Dhama et al., 2020; Spurgers et al., 2008). In this case, antisense oligonucleotides (ASOs) targeting specific sequences on mRNAs, small RNAs, or long non-coding RNAs enter the cell following an unclear mechanism. They recognize and hybridize specifically to targets based on Watson-Crick base pairing which results in the hetero-duplex formation (ASO/target RNA). At this point, hetero-duplex structures lead to suppression of translation through either by preventing ribosomal assembly at the 5' cap directly or cleavage by RNase H (Figure 1) (Aartsma-Rus et al., 2005; Crooke, 2017; Frazier, 2015; Hendling and Barišić, 2019; Miller and Harris, 2016; Spurgers et al., 2008; Xu et al., 2018). For instance, miravirsen (SPC3649) is an experimental antisense drug used for the treatment of hepatitis $\mathrm{C}$ by targeting human miRNA, miR-122 which protects HCV RNA against nuclease degradation, and 15mer ASO is already in Phase II clinical trials as of 2017 (Jonathan et al., 2014; Sigma-Aldrich, 2020). Such studies on antisense therapy are accumulating and will be developed further for the treatment of viral infections in the future with the help of its low toxicity, high specificity, and low production cost (Barrey et al., 2020). In the case of SARS-CoV-2 treatment, SARS-CoV-2 genomic RNA can be targeted by ASOs and block the translation of viral particles upon incorrect viral RNA processing. Therefore, antisense therapy could be also a promising approach to treat viral infections specifically through the inhibition of viral genome activity (Spurgers et al., 2008).

In this study, I aimed to design promising ASOstargeting SARS-CoV-2 to inhibit viral genome replication and transcription. Studies on fomivirsen, mipomersen, and miravirsen, antisense drugs, were taken as reference works to design this idea. To this purpose, 5 'UTR, $3^{\prime}$ UTR, and start codon in the SARS-CoV2 genomic RNA were analyzed to find out the regions without secondary structures. ASOs were then designed by targeting those available regions. All ASO candidates were evaluated through in vitro and in 
vivo tested parameters by Aartsma-Rus et al. and screened for off-targets. In the final stage, modifications required for functionality were determined for each promising ASOs.

\section{Materials and Methods}

\subsection{ASO design}

The reference sequence of SARS-CoV-2 genomic RNA (GenBank: MT385474) was used to design ASO candidates (NCBI, 2020). The design was primarily based on targeting 5'UTR, 3`UTR, and start codon (Figure 2). In particular, ASO candidates were targeting an open region in the secondary structure of the SARS-CoV-2 genomic RNA as predicted by m-fold for each targeting site. ASO candidates were scored through the parameters as described by Aartsma-Rus et al. previously. In this purpose, Tm values, ASO length, molecular weight, number, and percentage of GCs nucleotides, secondary structure formation, and dimer formation were calculated with the Oligonucleotide Properties Calculator tool (Oligo Calc, 2020). All ASO candidates were scored based on these parameters, only high-scored ASOs were included in the further analysis.

\subsection{ASO analysis}

Off-target risks for high-scored ASOs were evaluated on the GenBank database by using the BLAST tool. ASO candidates with a high similarity rate $($ E-value $<10)$ on the human genome database were eliminated. Free energy values were used to calculate the binding energy of ASO-target complexes. In this context, free energy values for ASO-target complexes and free energy for SARS-CoV-2 genomic RNA were determined on RNAstructure server (version 4.5). The binding energy of ASO-target complexes was identified using the equation below;

Binding energy of ASO/target complex $=$ Free energy ${ }_{\text {ASo/target complex }}-$ Free energy target $_{\text {ta }}$

ASO candidates with a higher binding energy of ASO-target complexes than $-20 \mathrm{kcal} / \mathrm{mol}$ were also eliminated since they will be probably inefficient as described by Aartsma-Rus et al. previously.

\subsection{ASO modifications}

Lastly, promising ASOs were then modified to improve their efficiency in the cell. All these modifications were determined and conducted based on literature.

\section{Results and Discussion}

To design antisense oligonucleotides (ASOs) targeting SARS-CoV-2 genomic RNA, I obtained firstly reference sequence of SARS-CoV-2 genomic RNA (GenBank; MT385474). ASOs are chemically modified oligonucleotides with base-complementary abilities to a specific RNA target in the cell. ASOs designed near to 5'UTR, start codon, and or 3'UTR of the targeted gene can regulate the translation of genetic material into functional proteins. Particularly, the suppression of translation is achieved through a translational blockage or recruiting sequence-specific nucleases in a sequence-specific manner upon binding of ASOs to complementary RNA (Liang et al., 2017). Thus, secondary structures of three viral regions on SARS-CoV-2 genomic RNA; 5 UTR, start codon, and or 3'UTR as the target in ASO design 
were predicted using mfold server to detect theoretical open regions available for ASO complementary. Two different conformations with the lowest free energy were taken as the template for each target site, and 24 ASO candidates targeting the open regions were designed in total (Supplementary Table 1-2).

I picked up the seven ASOs with a high potential to inhibit SARS-CoV-2 replication and transcription by inducing cleavage of the viral genomic RNA among 24 ASO candidates based on the parameters as described by Aartsma-Rus et al. previously (Figure 4-6). All these seven ASOs (Table 1) have 20-24 nucleotides (nt) in length which is an optimum size for synthesis and long enough on statistical grounds to specify in the human genome. Moreover, previous studies showed that the basic melting temperature (Tm) was significantly higher for the effective group of ASOs. Thereby, Tm values for ASOs were higher than $50^{\circ} \mathrm{C}$ in high-scored ASOs as well. Also, it is known that $\mathrm{C}$ and $\mathrm{G}$ content correlates with ASO-target RNA duplex stability, and the total number of Gs and Cs was significantly higher in effective ASOs. Therefore, I kept C and G content more than 6 for each high-scored ASOs or more than $30 \%$ in particular. I also screened high-scored ASOs in the case of the ability to form stable secondary structures and dimerization since it has a negative effect on ASO efficacy and eliminated them. Because dimers and secondary structures will probably decrease ASO efficacy by blocking their complementary with the target (Aartsma-Rus et al., 2009).

Off-target risks on the human transcriptome were checked through the BLAST tool. The ASOs with high similarity with any gene on the human transcriptome were left out of the list to avoid possible side effects due to the antisense therapy. Then, high-scored ASOs with a low off-target risks were handled for further modifications.

Natural phosphodiester oligonucleotides are quickly digested by cellular nucleases in the cell (Djavanbakht Samani et al., 2001). Therefore, ASOs were modified to some forms that are nucleaseresistant and appropriate for research purposes (Table 2). Several modifications such as 5-methyl deoxycytosine (5Me-dC) deaza G (7-deaza-dG) 2'-O-methoxy-ethyl 5me Uridine (2'-O-MOE-RNA) phosphorothioate linkage (PS) have been applied for high-scored ASOs to modify them through a research purpose and more resistant to cellular nucleases (Spurgers et al., 2008; Urban and Noe, 2003).

PS that link nucleotides through a phosphate backbone variant facilitates ASO entry into the cell since they retain negative backbone charges. Moreover, PS linkages are nuclease resistant, providing longer plasma half-lives in the cell. On the contrary, they have several negative impacts on ASO activity such as reduced hybridization to target mRNAs, phosphorothioate interaction with proteins, therefore lead to negative side effects, including immune system activity. For this purpose, all ASOs are modified with PS internucleotide linkages to protect them from cellular nucleases (Behlke et al., 2005) (Figure 7a).

2'-O-MOE-RNA (MOE is 2-methoxyethyl) is a type of enhanced-binding-affinity sugar modification. ASOs containing 2'-MOE modifications have enhanced nuclease resistance with lower cell toxicity and increased binding affinity to the target sequences. However, ASOs fully including sugar-modified RNAlike nucleotides (such as 2'-MOE) do not support RNase H cleavage of the complementary RNA, therefore ASOs should be constructed in the hetero form to induce RNase H cleavage. In this case, TR_1,3, and 7 were modified by 2'-MOE modifications to increase binding affinity and decrease cell toxicity (Behlke et al., 2005) (Figure 7c). 
Also, ASOs involving unmethylated $\mathrm{CpG}$ islands may stimulate the immune system like that of bacterial DNA as the CpG motifs in the bacterial genome triggers the activation of immune cells. To get rid of immunostimulation, designed ASOs should not include a high amount of $\mathrm{CpG}$ islands or if it involves, it must be modified the cytosines in $\mathrm{CpG}$ with 5'-methylcytosine, which has been shown to decrease immunostimulation significantly. TR_2, 4, 6 which have at least one $\mathrm{CpG}$ island were modified by replacing cytosines with 5'-methylcytosine (Behlke et al., 2005) (Figure 7d).

Lastly, ASOs containing two or more $\mathrm{C}$ or $\mathrm{G}$ nucleotides can form inappropriate structures, which might trigger undesirable, off-target effects. Previous studies have shown that polyG stretches of $\mathrm{G}$ bases are able to lead the formation of G-quartets, which may help binding to proteins, by the way, transcription factors, thereby interfere with antisense activity. To avoid G-quartets, 7-deaza-dG modifications to replace $\mathrm{G}$ bases which will probably prevent quartet formation. PolyG including TR_3, 4, and 5 ASOs undergone modifications to replace $\mathrm{G}$ bases to 7-deaza-dG modified forms (Behlke et al., 2005) (Figure 7b).

In the final stage of the study, I evaluated the binding energy of ASO-target complexes to identify their efficiency in silico (Aartsma-Rus et al., 2009). To do this, free energy values were used to calculate the binding energy of AON-target complexes as described in the methods. Aartsma-Rus at el. previously demonstrated that the binding energy of ASO-target complexes was significantly higher for effective ASOs $(<-20$, respectively). In this line, our results showed that all ASOs have less binding free energy than $-20 \mathrm{kcal} / \mathrm{mol}$ (Figure 8 ).

Overall, this study hidhlighted that antisense theraphy could be a novel strategy to threat present COVID19 pandemic by targeting SARS-CoV-2 genomic RNA directly. In this context, ASOs targeting 5 UTR, start codon, and or 3'UTR of the targeted gene were designed and eliminated low-scored ASO candidates. Seven high-scored ASOs based on parameters described in the literature were determined as a novel for future studies. Those seven promising ASOs can be synthesized followed by required modifications and test on SARS-CoV-2 infected Vero cells to screen their efficacy for the inhibition of viral replication and transcription (Yao et al., 2020).

\section{Acknowledgements}

I would like to thank Ozlem Yalcin Capan, Asst. Prof. for assistance with her guide during paper publishing, and for her comments that greatly improved the manuscript. I would also like to show my gratitude to the Nazlican Yurekli, BSc for sharing her pearls of wisdom with me during the course of this research. I am also immensely grateful to my family for their support.

This research did not receive any specific grant from funding agencies in the public, commercial, or notfor-profit sectors.

\section{References}

1. Aartsma-Rus A, De Winter CL, Janson AAM, Kaman WE, Van Ommen GJB, Den Dunnen JT, et al. Functionalanalysis of 114 exon-internal AONs for targeted DMD exon skipping: Indication for steric hindrance of SR protein binding sites. Oligonucleotides 2005;15:284-97. https://doi.org/10.1089/oli.2005.15.284. 
2. Aartsma-Rus A, van Vliet L, Hirschi M, Janson AAM, Heemskerk H, de Winter CL, et al. Guidelines for antisense oligonucleotide design and insight into splice-modulating mechanisms. Mol Ther 2009;17:548-53. https://doi.org/10.1038/mt.2008.205.

3. Barrey E, Burzio V, Dhorne-pollet S, Delmas B. Think Different with RNA Therapy : Can Antisense Oligonucleotides Be Used to Inhibit Replication and Transcription of SARS-Cov-2? PrepintsOrg 2020:1-23. https://doi.org/10.20944/preprints202004.0412.v1.

4. Behlke MA, Devor EJ, Goodchild J. Designing Antisense Oligonucleotides 2005.

5. Crooke ST. Molecular Mechanisms of Antisense Oligonucleotides. Nucleic Acid Ther 2017;27:70-7. https://doi.org/10.1089/nat.2016.0656.

6. Cubuk H, Ozbil M. Comparison of Clinically Approved Molecules on SARS-CoV-2 Drug Target Proteins: A Molecular Docking Study. PrepintsOrg 2020. https://doi.org/10.26434/CHEMRXIV.12090828.V2.

7. Dhama K, Sharun K, Tiwari R, Dadar M, Malik YS, Singh KP, et al. COVID-19, an emerging coronavirus infection: advances and prospects in designing and developing vaccines, immunotherapeutics, and therapeutics. Hum Vaccines Immunother 2020;16:1232-8. https://doi.org/10.1080/21645515.2020.1735227.

8. Dja vanbakht Samani T, Jolles B, Laigle A. Best minimally modified antisense oligonucleotides according to cell nuclease activity. Antisense Nucleic Acid Drug Dev 2001;11:129-36.https://doi.org/10.1089/108729001300338654.

9. Frazier KS. Antisense Oligonucleotide Therapies:The Promise and the Challenges from a Toxicologic Pathologist's Perspective. Toxicol Pathol2015;43:78-89. https://doi.org/10.1177/0192623314551840.

10. Fung TS, Liu DX. Human coronavirus: Host-pathogen interaction. Annu Rev Microbiol 2019;73:529-57. https://doi.org/10.1146/annurev-micro-020518-115759.

11. Genelink.com. oligos - custom oligo synthesis, oligonucleotides, custom rna synthesis, gel page rpc hplc reverse cartridge purification,modifications,rnai sirna,probes from Gene Link 2020. http://www.genelink.com/ (accessed August 9, 2020).

12. Hendling M, Barišić I. In-silico Design of DNA Oligonucleotides: Challenges and Approaches. Comput Struct Biotechnol J 2019;17:1056-65.https://doi.org/10.1016/i.csbj.2019.07.008.

13. Hoffmann M, Kleine-Weber H, Schroeder S, Krüger N, Herrler T, Erichsen S, et al. SARS-CoV-2 Cell Entry Depends on ACE2 and TMPRSS2 and Is Blocked by a Clinically Proven Protease Inhibitor. Cell 2020;181:271-280.e8. https://doi.org/10.1016/j.cell.2020.02.052.

14. Jeong GU, Song H, Yoon GY, Kim D, Kwon Y-C. Therapeutic Strategies against COVID-19 and Structural Characterization of SARS-CoV-2: A Review. Front Microbiol 2020;11:1723. https://doi.org/10.3389/FMICB.2020.01723.

15. Jonathan LFR, Rebhan GMAE, Crivelli SEM, Rémy D, Stoffel M, Hall J. Mira virsen (SPC3649) can inhibit the biogenesis of miR-122. Nucleic Acids Res 2014;42:609-621.https://doi.org/https://doi.org/10.1093/nar/gkt852.

16. Liang XH, Sun H, Nichols JG, Crooke ST. RNase H1-Dependent Antisense Oligonucleotides Are Robustly Active in Directing RNA Cleavage in Both the Cytoplasm and the Nucleus. Mol Ther 2017;25:2075-92. https://doi.org/10.1016/i.ymthe.2017.06.002.

17. Miller CM, Harris EN. Antisense Oligonucleotides: Treatment Strategies and Cellular Internalization. RNA Dis 2016;3:e1393. https://doi.org/10.14800/rd.1393.

18. NCBI. Severe acute respiratory syndrome coronavirus 2 isolate SARS-CoV-2/hum - Nucleotide - NCBI 2020. https://www.ncbi.nlm.nih.gov/nuccore/MT385474 (accessed July 30, 2020).

19. Oligo Calc. OligoCalc: $\quad$ Oligonucleotide $\quad$ Properties 2020. http://biotools.nubic.northwestern.edu/OligoCalc.html(accessed July 30, 2020).

20. Shang J, Wan Y, Luo C, Ye G, Geng Q, Auerbach A, et al. Cell entry mechanisms of SARS-CoV-2. Proc Natl Acad Sci U S A 2020;117:11727-34.https://doi.org/10.1073/pnas.2003138117.

21. Sigma-Aldrich. Antisense Oligonucleotides | Sigma-Aldrich 2020. https://www.sigmaaldrich.com/technicaldocuments/articles/biology/antisense-oligonucleotides.html\#ref (accessed July 30, 2020).

22. Smith T, Bushek J, Prosser T. COVID-19 Drug Therapy - PotentialOptions. Elsevier 2020.

23. Spurgers KB, Sharkey CM, Warfield KL, Bavari S. Oligonucleotide antiviral therapeutics: Antisense and RNA interference for highly pathogenic RNA viruses. Antiviral Res 2008;78:26-36. https://doi.org/10.1016/i.antiviral.2007.12.008.

24. Urban E, Noe CR. Structural modifications of antisense oligonucleotides. Farmaco 2003;58:243-58. https://doi.org/10.1016/S0014-827X(03)00022-3.

25. Walls AC, Park YJ, Tortorici MA, Wall A, McGuire AT, Veesler D. Structure, Function, and Antigenicity of the SARSCoV-2 Spike Glycoprotein. Cell 2020;181:281-292.e6.https://doi.org/10.1016/j.cell.2020.02.058.

26. Worldometer. Corona virus Cases. Worldometer 2020:1-22.https://doi.org/10.1101/2020.01.23.20018549V2.

27. Xu J zhong, Zhang J lan, Zhang W guo. Antisense RNA: the new favorite in genetic research. J Zhejiang Univ Sci B 2018;19:739-49. https://doi.org/10.1631/jzus.B1700594. 
28. Yan R, Zhang Y, Li Y, Xia L, Guo Y, Zhou Q. Structural basis for the recognition of SARS-CoV-2 by full-length human ACE2. Science (80- ) 2020;367:1444-8. https://doi.org/10.1126/science.abb2762.

29. Yao X, Ye F, Zhang M, Cui C, Huang B, Niu P, et al. In Vitro Antiviral Activity and Projection of Optimized Dosing Design of Hydroxychloroquine for the Treatment of Severe Acute Respiratory Syndrome Coronavirus 2 (SARS-CoV-2) | Publons 2020. https://publons.com/publon/30584975/ (ac cessed July 31, 2020). 


\section{Figures}

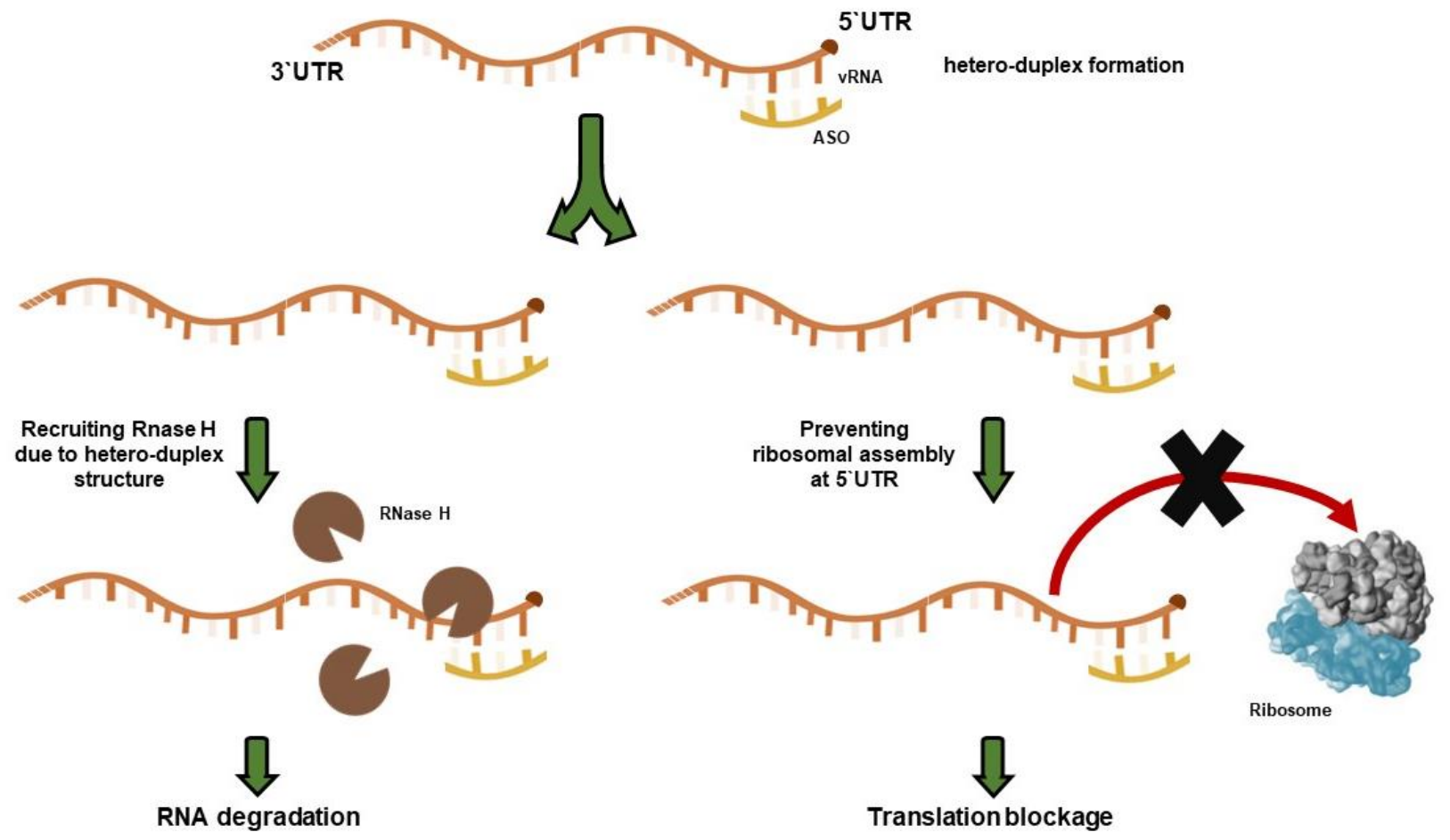

Figure 1: The principle of antisense RNA therapy. ASOs targeting specific sequences on viral genomic RNA enter the cell through an unclear mechanism. They recognize and hybridize specifically to targets based on Watson-Crick base pairing which results in the hetero-duplex formation (ASO/target RNA). At this point, hetero-duplex structures lead to suppression of translation through either by preventing ribosomal assembly at the 5' cap directly or cleavage by RNase H. 


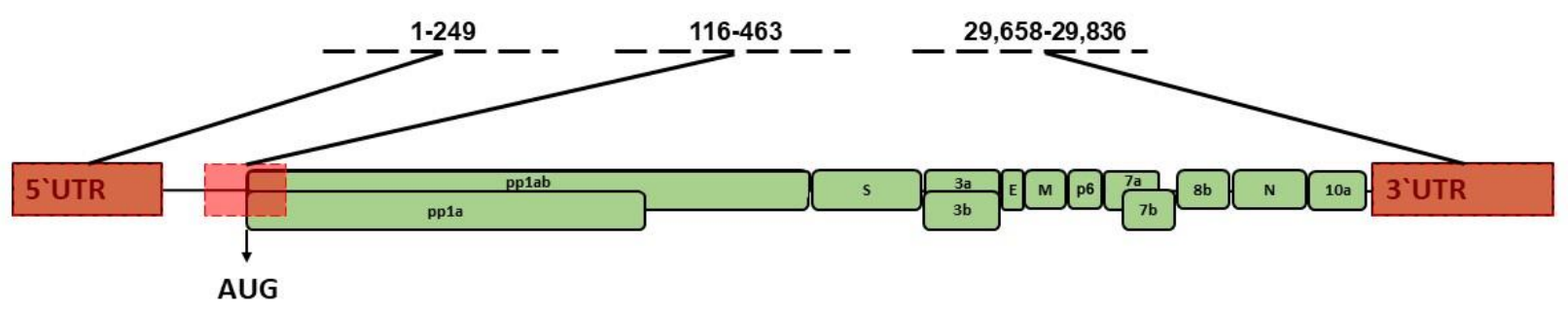

Figure 2: Locations targeted by ASOs on SARS-CoV-2 genomic RNA; 5 UTR (1-249 bases), 3'UTR (116-463 bases), and start codon (29,658-29,836 bases).

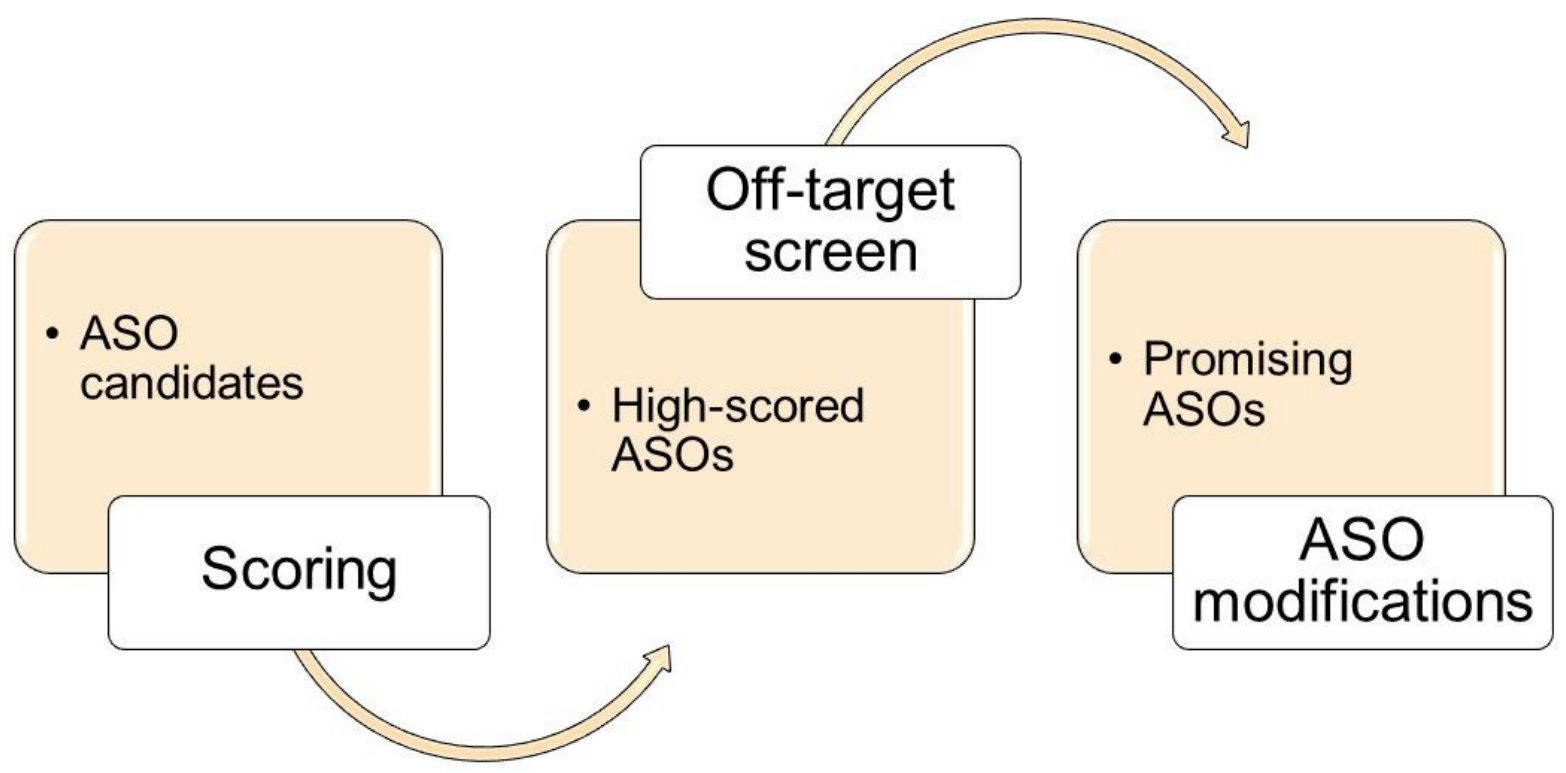

Figure 3: Design algorithm. ASO candidates primarily based on targeting 5 UTR, 3`UTR, and start codon were scored. High-scored ASOs were screened for possible off-targets. Promising ASOs after off-target screening were modified through the ASOs`purposes. 


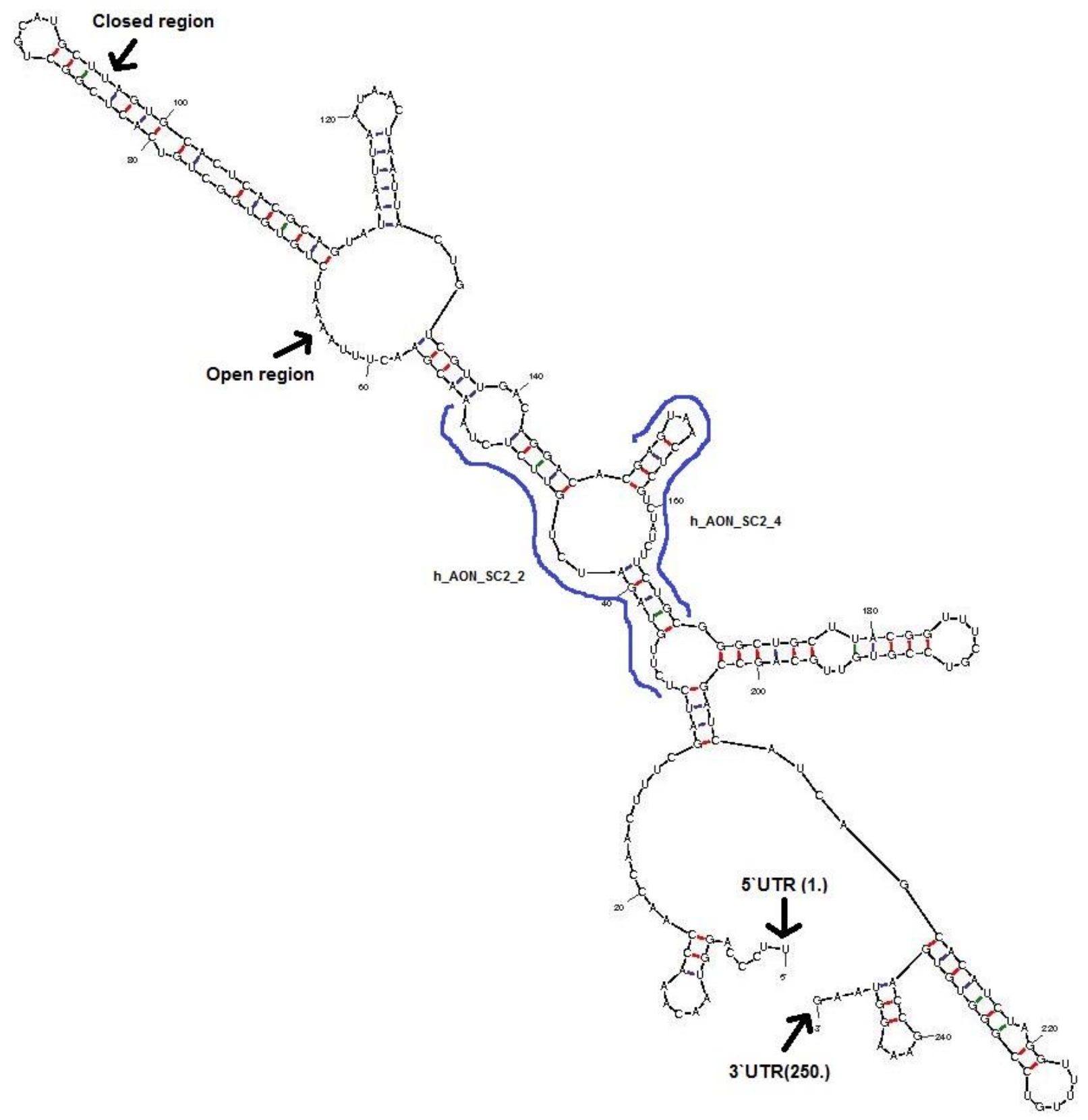

Figure 4a: Predicted second ary structure for $5 \mathrm{UTR}(\mathrm{dG}=-72.12)$ and representation of designed ASOs. 


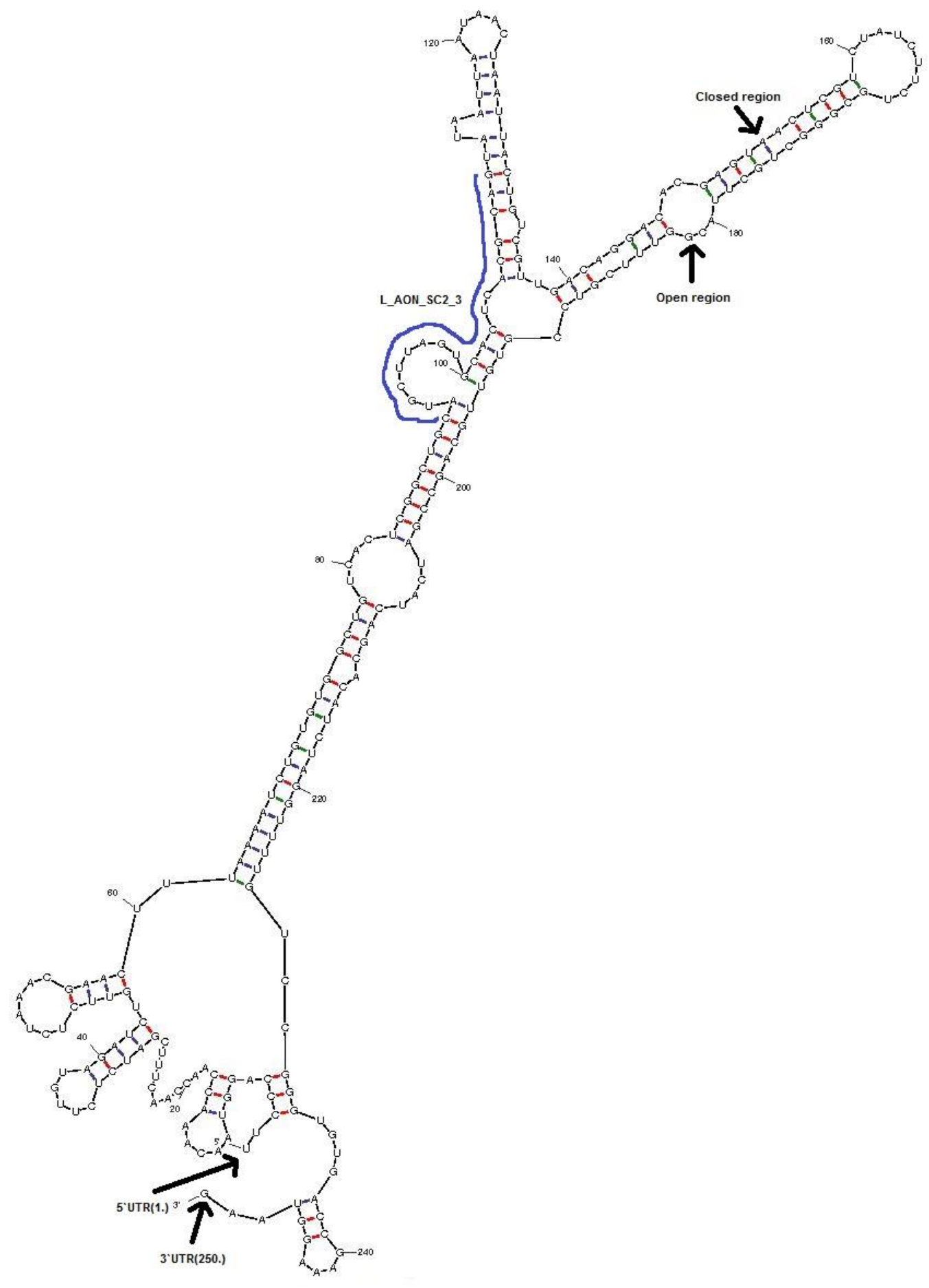

Figure 4b: Predicted secondary structure for 5 UTR $(\mathrm{dG}=-70.19)$ and representation of designed ASOs. 


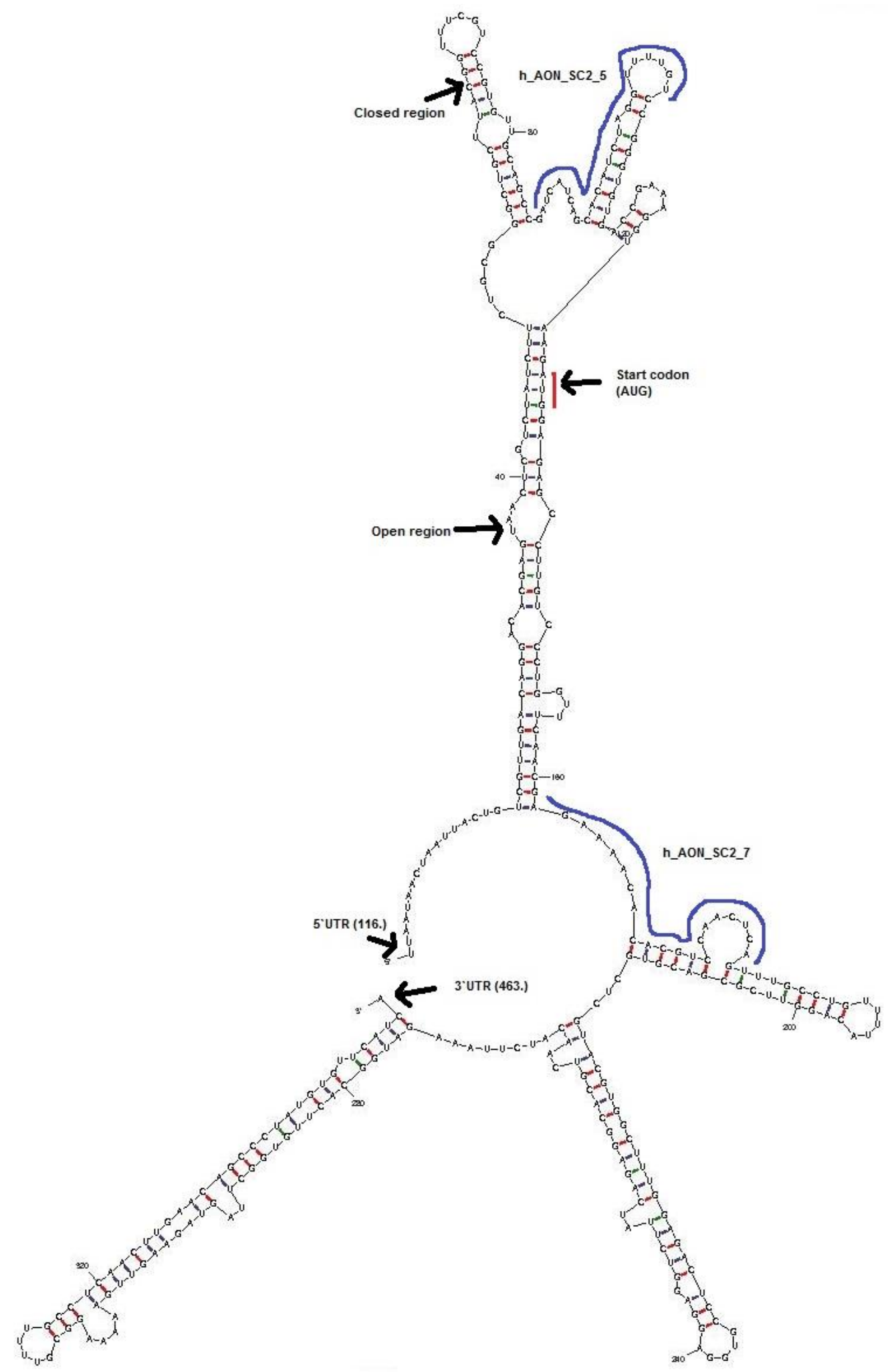

Figure 5: Predicted secondary structure for start codon region $(\mathrm{dG}=-105.00)$ and representation of designed ASOs. 


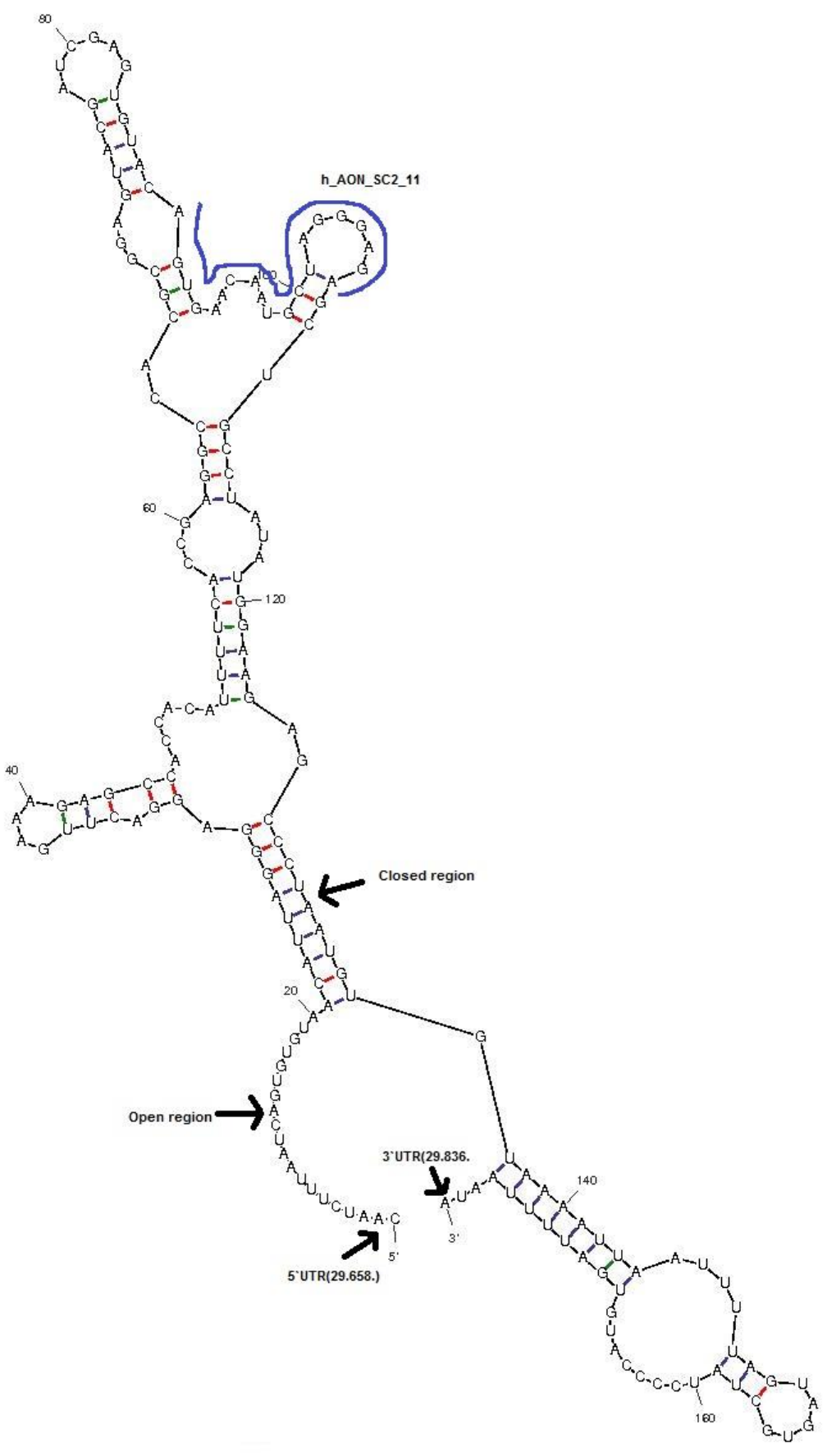

Figure 6a: Predicted secondary structure for $3{ }^{\prime} \mathrm{UTR}(\mathrm{dG}=-33.66)$ and representation of designed ASOs. 


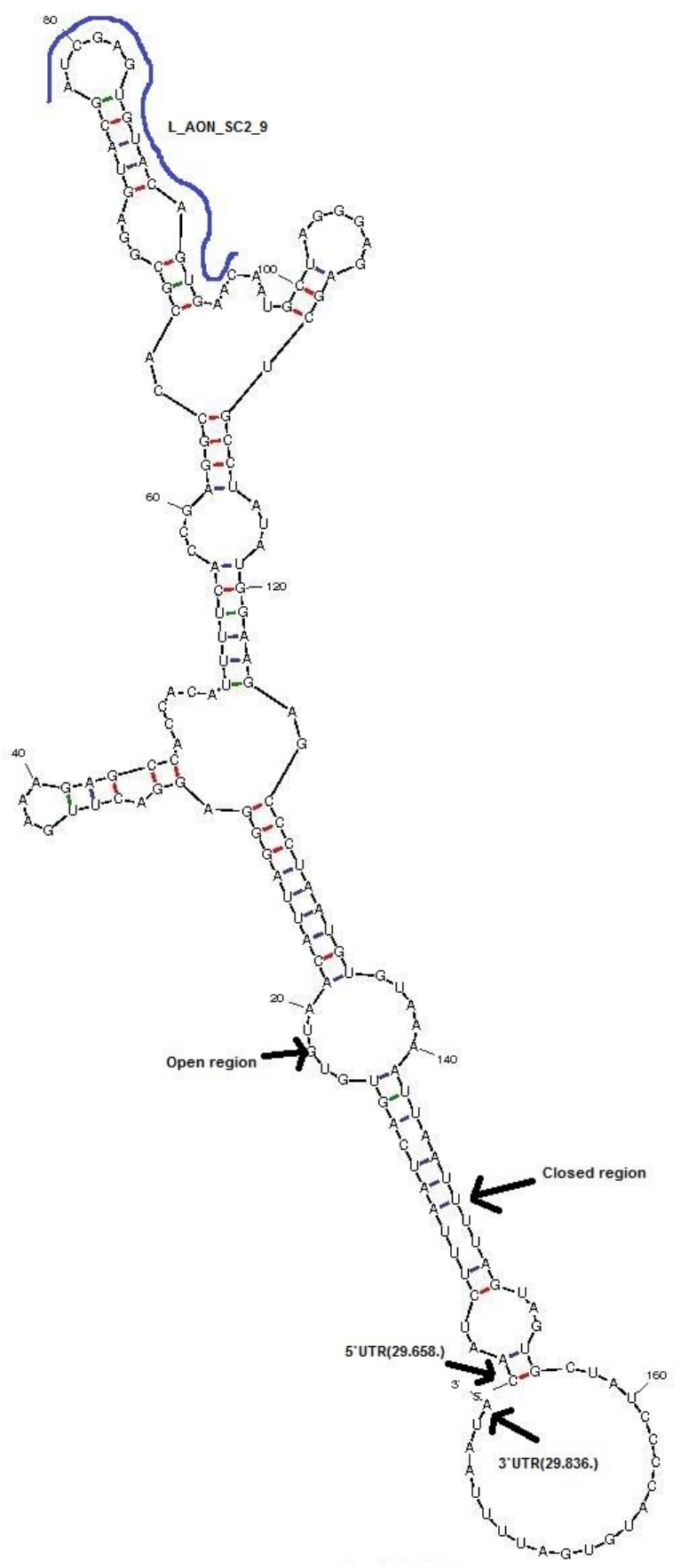

Figure 6b: Predicted secondary structure for 3`UTR $(\mathrm{dG}=-31.10)$ and representation of designed ASOs. 


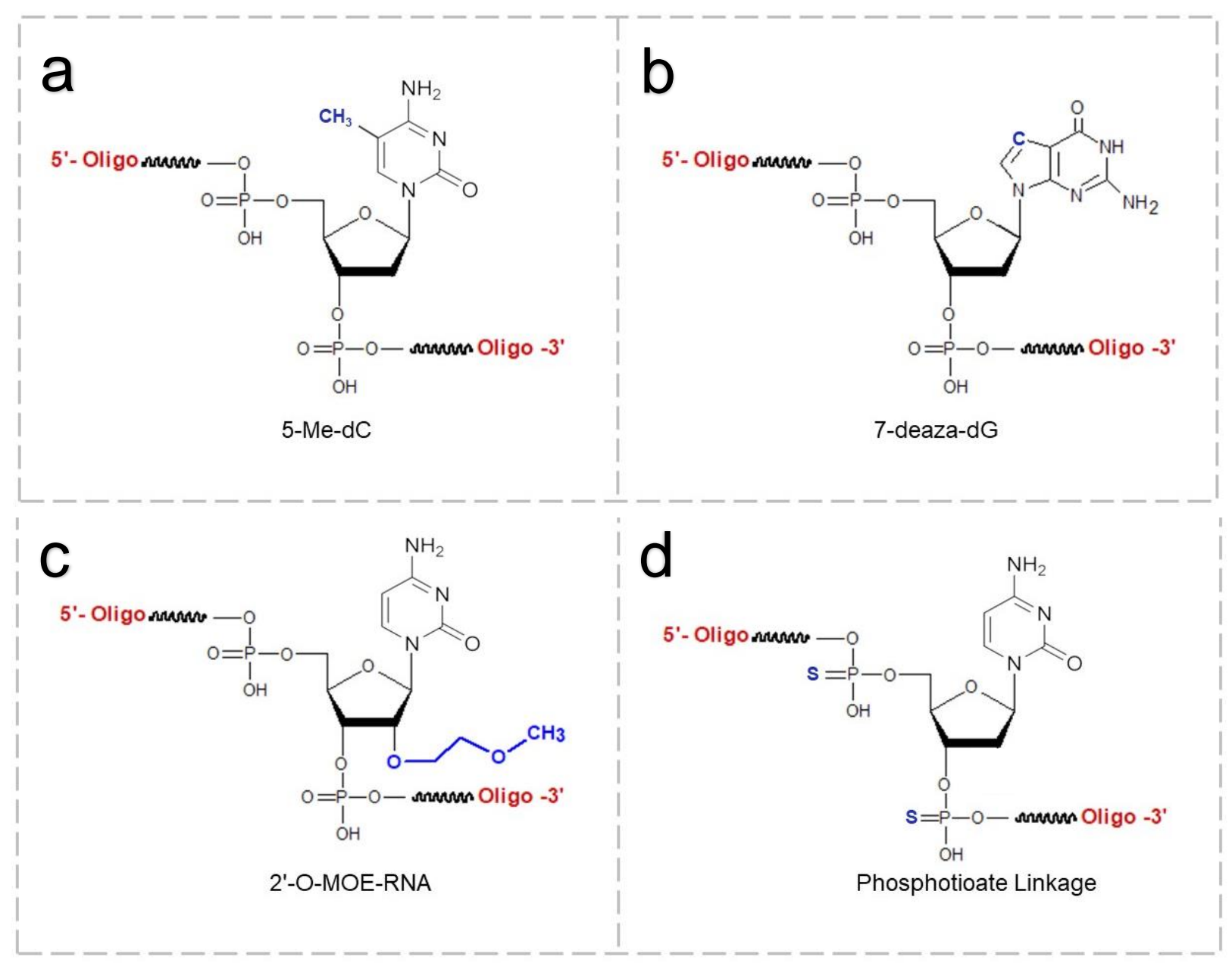

Figure 7: ASO modifications. (a) 5-methyl deoxycytosine (5Me-dC). (b) deaza G (7-deaza-dG). (c) 2'-O-methoxy-ethyl 5me Uridine (2'-O-MOE-RNA). (d) Phosphorothioate linkage (PS) (Genelink.com 2020). 


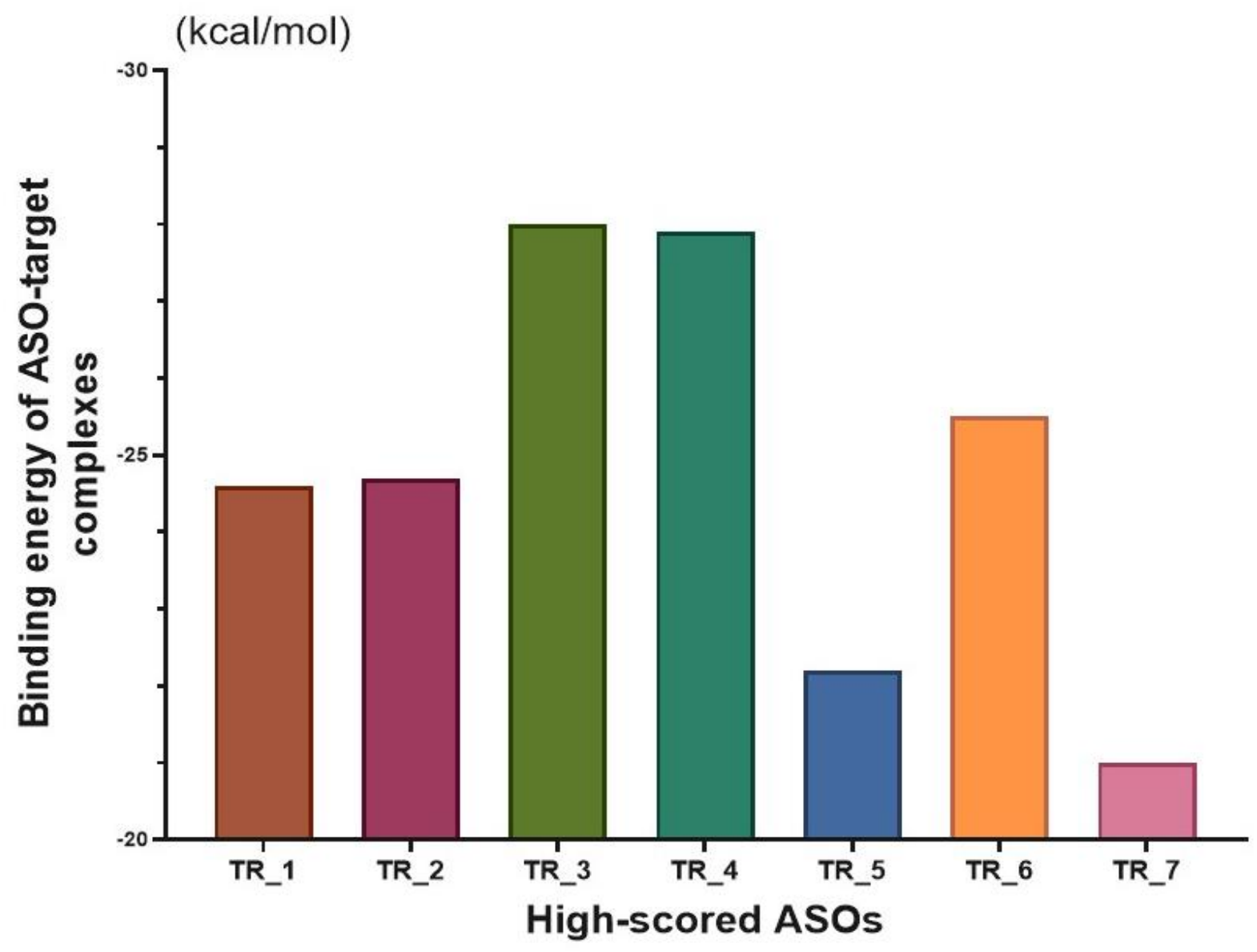

Figure 8: Binding energy values for high-scored ASOs. TR_3 and TR_4 have the highest binding energy, approximately $-28 \mathrm{kcal} / \mathrm{mol}$ particularly. 
Table 2: Antisense oligonucleotides with high-score in modified forms.

\begin{tabular}{|c|c|}
\hline Name & Modified structures \\
\hline TR_1 & $\underline{U^{*} U^{*} A^{*} G^{*} A^{*} G^{*} A^{*} A^{*} C^{*} A^{*} G^{*} A^{*} U^{*} C^{*} U^{*}{ }^{*} C^{*} A^{*} A^{*} G^{*} A}$ \\
\hline TR_2 & $C^{*} A^{*} G^{*} A^{*} A^{*} G^{*} A^{*} U^{*} A^{*} G^{*} A^{*} m C^{*} G^{*} A^{*} G^{*} U^{*} U^{*} A^{*} C^{*} U$ \\
\hline TR_3 & $\underline{A^{*} C^{*} A^{*} A^{*} A^{*} A^{*}} \times C^{*} x C^{*} U^{*} A^{*} G^{*} A^{*} U^{*} G^{*} U^{*} G^{*} C^{*} U^{*} G^{*} A^{*} U^{*} G^{*} A^{*} U$ \\
\hline TR_4 & $C^{*} U^{*} G^{*} A^{*} G^{*} U^{*} U^{*} x G^{*} x G^{*} A^{*} m C^{*} G^{*} U^{*} G^{*} U^{*} G^{*} U^{*} U^{*} U^{*} U^{*} C^{*} U$ \\
\hline TR_5 & $U^{*} C^{*} U^{*} x C^{*} x C^{*} x C^{*} U^{*} A^{*} G^{*} C^{*} A^{*} U^{*} U^{*} G^{*} U^{*} U^{*} C^{*} A^{*} C^{*} U$ \\
\hline TR_6 & $C^{*} U^{*} G^{*} m C^{*} G^{*} U^{*} G^{*} A^{*} G^{*} U^{*} G^{*} C^{*} A^{*} C^{*} U^{*} A^{*} A^{*} G^{*} C^{*} A^{*} U$ \\
\hline TR_7 & $\underline{G^{*} U^{*} U^{*} C^{*} A^{*} C^{*} U^{*} G^{*} U^{*} A^{*} C^{*} A^{*} C^{*} U^{*} m C^{*} G^{*} A^{*} U^{*} m C^{*} G}$ \\
\hline
\end{tabular}

$$
\begin{aligned}
& \text { Underline }=2^{\prime}-O-M O E-R N A \text { (MOE is 2-methoxyethyl) } \\
& \mathrm{m}=5-\mathrm{Me}-\mathrm{dC} \\
& *=\mathrm{PS} \\
& \mathrm{x}=7 \text {-deaza-dG }
\end{aligned}
$$




\section{Highlights}

- Low toxicity, high specificity, and low cost highlight antisense based therapies.

- Antisense therapy is a promising approach to prevent SARS-CoV-2 infection.

- ASOs targeting SARS-CoV-2 genome can inhibit viral translation and replication 


\section{Graphical Abstract}

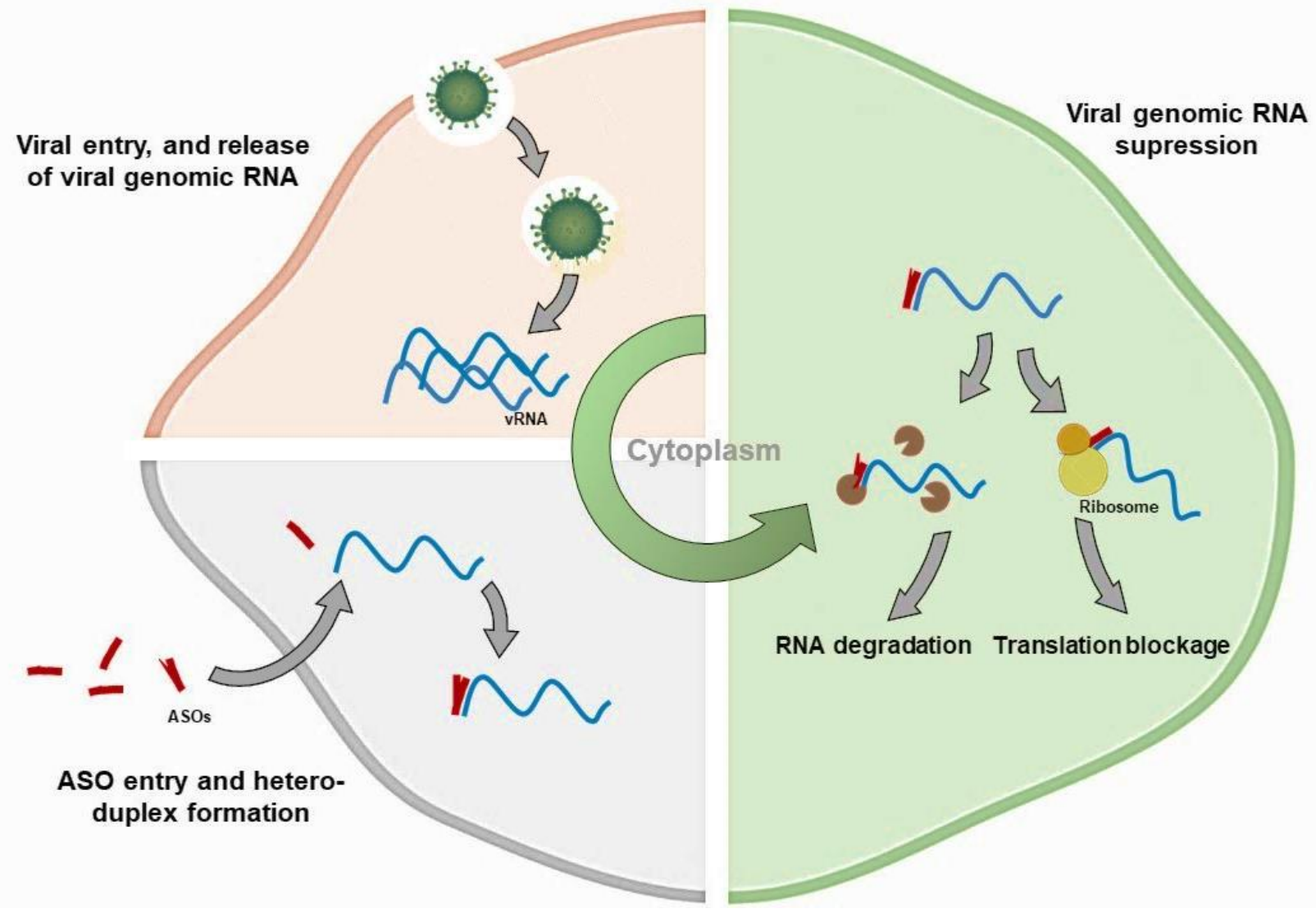


Tables

Table 1: High-scored antisense oligonucleotides based on the parameters described previously.

\begin{tabular}{|c|c|c|c|c|c|c|c|c|c|c|c|}
\hline AONs & Name & $\begin{array}{l}\text { Sense Sequence } \\
\left(5^{\prime}->3^{\prime}\right)\end{array}$ & $\begin{array}{l}\text { Antisense Sequence } \\
\left(5^{\prime}->3^{\prime}\right)\end{array}$ & $\begin{array}{l}\text { Target } \\
\text { region }\end{array}$ & Length & Mw & $\mathrm{Tm}\left({ }^{\circ} \mathrm{C}\right)$ & GCs & GC\% & $\begin{array}{l}\text { Secondary } \\
\text { Structure }\end{array}$ & Dimer \\
\hline h_AON_SC2_2 & TR_1 & TCTTGTAGATCTGTTCTCTAA & UUAGAGAACAGAUCUACAAGA & 5UTR & 21 & 6751,2 & 52,8 & 7 & 33,3 & Weak & No \\
\hline h_AON_SC2_4 & TR_2 & AGTAACTCGTCTATCTTCTG & CAGAAGAUAGACGAGUUACU & 5UTR & 20 & 6438 & 53,5 & 8 & 40 & None & No \\
\hline h_AON_SC2_5 & TR_3 & ATCATCAGCACATCTAGGTITTGT & ACAAAACCUAGAUGUGCUGAUGAU & Startcodon & 24 & 7684,7 & 59,6 & 9 & 37,5 & Very Weak & No \\
\hline h_AON_SC2_7 & TR_4 & AGAAAACACACGTCCAACTCAG & CUGAGUUGGACGUGUGUUUUCU & Startcodon & 22 & 6990,1 & 59,4 & 10 & 45,5 & None & No \\
\hline h_AON_SC2_11 & TR_5 & AGTGAACAATGCTAGGGAGA & UCUCCCUAGCAUUGUUCACU & 3`UTR & 20 & 6201,7 & 59,2 & 9 & 45 & None & No \\
\hline L_AON_SC2_3 & TR_6 & ATGCTTAGTGCACTCACGCAG & CUGCGUGAGUGCACUAAGCAU & 5 UTR & 21 & 6712,1 & 63,1 & 11 & 52,4 & Weak & No \\
\hline L_AON_SC2_9 & TR_7 & ATCGAGTGTACAGTGAAC & GUUCACUGUACACUCGAUCG & 3`UTR & 20 & 6303,8 & 57,1 & 10 & 50 & None & No \\
\hline
\end{tabular}




\section{Supplementary Figures}

Table 1: ASO candidates.

\begin{tabular}{|c|c|c|c|c|c|c|c|c|c|c|c|}
\hline AONs & $\begin{array}{l}\text { Sense Sequence } \\
\qquad\left(5^{\prime}->3^{\prime}\right)\end{array}$ & $\begin{array}{c}\text { Antisense Sequence } \\
\qquad\left(5^{\prime}->3^{\prime}\right)\end{array}$ & $\begin{array}{l}\text { Target } \\
\text { region }\end{array}$ & $\begin{array}{l}\text { Off-targets } \\
(\text { E-value }<10)\end{array}$ & $\begin{array}{c}\text { Length } \\
\text { (base) }\end{array}$ & Mw & $\operatorname{Tm}\left({ }^{\circ} \mathrm{C}\right)$ & GCs & GC\% & $\begin{array}{l}\text { Secondary } \\
\text { Structure }\end{array}$ & Dimer \\
\hline h_AON_SC2_1 & AGGTAACAAACCAACCAACTTT & AAAGUUGGUUGGUUUGUUACCU & 5UTR & NR_038987 & 22 & 6998,2 & 57,4 & 8 & 36,4 & Weak & No \\
\hline h_AON_SC2_2 & TCTTGTAGATCTGTTCTCTAA & UUAGAGAACAGAUCUACAAGA & 5UTR & - & 21 & 6751,2 & 52,8 & 7 & 33,3 & Weak & No \\
\hline h_AON_SC2_3 & CTAAACGAACTTTAAAATCT & AGAUUUUAAAGUUCGUUUAG & 5UTR & $\begin{array}{l}\text { NM_001288623.1,NM_0012 } \\
\text { 88622.1, NM_138468.5 }\end{array}$ & 20 & 6354,8 & 43,7 & 5 & 25 & Very Weak & No \\
\hline h_AON_SC2_4 & AGTAACTCGTCTATCTTCTG & CAGAAGAUAGACGAGUUACU & 5UTR & - & 20 & 6438 & 53,5 & 8 & 40 & None & No \\
\hline h_AON_SC2_5 & ATCATCAGCACATCTAGGTTTTGT & ACAAAACCUAGAUGUGCUGAUGAU & Startcodon & - & 24 & 7684,7 & 59,6 & 9 & 37,5 & Very Weak & No \\
\hline h_AON_SC2_6 & TTAATAACTAATTACTGTCG & CGACAGUAAUUAGUUAUUAA & Startcodon & - & 20 & 6360,9 & 44,3 & 5 & 25 & Very Weak & Yes \\
\hline h_AON_SC2_7 & AGAAAACACACGTCCAACTCAG & CUGAGUUGGACGUGUGUUUUCU & Startcodon & - & 22 & 6990,1 & 59,4 & 10 & 45,5 & None & No \\
\hline h_AON_SC2_8 & TCAACATCTTAAAGATGGCA & UGCCAUCUUUAAGAUGUUGA & Startcodon & NM_018164.2,NM_021961.5 & 20 & 6329,8 & 53 & 7 & 35 & Moderate & No \\
\hline h_AON_SC2_9 & CAATCTTTAATCAGTGTGTAA & UUACACACUGAUUAAAGAUUG & 3'UTR & - & 21 & 6666,1 & 48,6 & 6 & 28,6 & None & No \\
\hline h_AON_SC2_10 & TGAAAGAGCCACCACATTTTCAC & GUGAAAAUGUGGUGGCUCUUUCA & 3'UTR & NM_181503.2 & 23 & 7365,4 & 60,5 & 10 & 43,5 & Weak & No \\
\hline h_AON_SC2_11 & AGTGAACAATGCTAGGGAGA & UCUCCCUAGCAUUGUUCACU & 3'UTR & - & 20 & 6201,7 & 59,2 & 9 & 45 & None & No \\
\hline h_AON_SC2_12 & ATTTAGTAGTGCTATCCCCATG & CAUGGGGAUAGCACUACUAAAAU & 3'UTR & - & 23 & 7378,5 & 59,4 & 9 & 39,1 & Very Weak & No \\
\hline
\end{tabular}


Table 2: ASO candidates.

\begin{tabular}{|c|c|c|c|c|c|c|c|c|c|c|c|}
\hline AONs & $\begin{array}{l}\text { Sense Sequence } \\
\qquad\left(5^{\prime}->3^{\prime}\right)\end{array}$ & $\begin{array}{l}\text { Antisense Sequence } \\
\qquad\left(5^{\prime}->3^{\prime}\right)\end{array}$ & $\begin{array}{l}\text { Target } \\
\text { region }\end{array}$ & $\begin{array}{l}\text { Off-targets } \\
(\text { E-value }<10)\end{array}$ & $\begin{array}{l}\text { Length } \\
\text { (base) }\end{array}$ & Mw & $\operatorname{Tm}\left({ }^{\circ} \mathrm{C}\right)$ & GCs & GC\% & $\begin{array}{l}\text { Secondary } \\
\text { Structure }\end{array}$ & Dimer \\
\hline L_AON_SC2_1 & AACCAACCAACTTTCGATCTCTTGTA & UACAAGAGAUCGAAAGUUGGUUGGUU & 5UTR & - & 26 & 8393,1 & 61,4 & 10 & 38,5 & None & No \\
\hline L_AON_SC2_2 & CTGTTCTCTAAACGAACTTT & AAAGUUCGUUUAGAGAACAG & 5'UTR & - & 20 & 6439 & 50,2 & 7 & 35 & Moderate & No \\
\hline L_AON_SC2_3 & ATGCTTAGTGCACTCACGCAG & CUGCGUGAGUGCACUAAGCAU & 5UTR & - & 21 & 6712,1 & 63,1 & 11 & 52,4 & Weak & No \\
\hline L_AON_SC2_4 & CGTCTATCTTCTGCGGGCTG & CAGCCCGCAGAAGAUAGACG & 5'UTR & $\begin{array}{l}\text { NM_001349551.1,NM_001349550.1, } \\
\text { NM_001349549.1 }\end{array}$ & 20 & 6451 & 63,8 & 12 & 60 & None & No \\
\hline L_AON_SC2_5 & TTAATAACTAATTACTGTCG & CGACAGUAAUUAGUUAUUAA & Startcodon & - & 20 & 6360,9 & 44,3 & 5 & 25 & Very Weak & Yes \\
\hline L_AON_SC2_6 & CGATCATCAGCACATCTAGG & CCUAGAUGUGCUGAUGAUCG & Startcodon & NM_182587.3, NM_032504.1, & 20 & 6383,9 & 58 & 10 & 50 & Very Weak & No \\
\hline L_AON_SC2_7 & GGTTCAACGAGAAAACACA & UGUGUUUUCUCGUUGAAACC & Startcodon & NM_001352011.1, NM_016145.3 & 20 & 6282,7 & 53,5 & 8 & 40 & Weak & No \\
\hline L_AON_SC2_8 & CAACATCTTAAAGATGGCACT & AGUGCCAUCUUUAAGAUGUUG & Startcodon & - & 21 & 6675 & 55 & 8 & 38,1 & Moderate & No \\
\hline L_AON_SC2_9 & ATCGAGTGTACAGTGAAC & GUUCACUGUACACUCGAUCG & 3'UTR & - & 20 & 6303,8 & 57,1 & 10 & 50 & None & No \\
\hline L_AON_SC2_10 & GAGGACTTGAAAGAGCCACC & GGUGGCUCUUUCAAGUCCUC & 3'UTR & $\begin{array}{l}\text { NM_000064.3,NM_004274.4, } \\
\text { NR_146581.1,NM_001303527.1 }\end{array}$ & 20 & 6296,8 & 62 & 11 & 55 & None & No \\
\hline L_AON_SC2_11 & TGTGTAAAATTAATTTTAGTA & UACUAAAAUUAAUUUUACACA & 3'UTR & $\begin{array}{l}\text { NM_001308116.1,NR_130138.1, } \\
\text { NM_006041.2, NM_024947.3 }\end{array}$ & 21 & 6595 & 40,8 & 3 & 14,3 & Very Weak & No \\
\hline L_AON_SC2_12 & GCTATCCCCATGTGA TTTTAAT & AUUAAAAUCACAUGGGGAUAGC & 3'UTR & $\begin{array}{l}\text { NR_039977.1, } \\
\text { NR_039975.1 }\end{array}$ & 22 & 7073,4 & 56,5 & 8 & 36,4 & Very Weak & No \\
\hline
\end{tabular}

